\title{
Challenges in the diagnosis and management of Cushing's syndrome due to ectopic ACTH from bronchial carcinoid
}

\author{
M S A Cooray ${ }^{1}$, N P Somasundaram ${ }^{1}$, Janakie Fernando², A H N Fernando ${ }^{3}$, R M De Silva ${ }^{2}$, \\ D Rasnayake ${ }^{4}$
}

Sri Lanka Journal of Diabetes, Endocrinology and Metabolism 2015; 5: 95-99

\begin{abstract}
Despite the advances in biochemical methods and imaging techniques, Cushing's syndrome (CS) related to ectopic adrenocorticotrophic hormone (ACTH) secretion continues to pose diagnostic and therapeutic challenges to the clinician. The work up involves establishment of endogenous Cushing'syndrone, diagnosis of ACTH dependency, localization of the source of ACTH secretion and rapid biochemical control of hypercortisolaemia. The diagnostic pathway is made difficult by recurrent sepsis, relative hypoadrenalism, drug side effects as well as unmasked incidental lesions.
\end{abstract}

We report a patient presenting with Cushing's syndrome associated with ectopic ACTH secretion from a bronchial carcinoid whose management presented multiple diagnostic and therapeutic challenges.

Key words: Cushing's syndrome, ectopic adrenocorticotrophic hormone, hypercortisolaemia, hypoadrenalism, incidental lesion, small cell carcinoma of lung.

\section{Introduction}

The syndrome of ectopic a ACTH secretion is one of the greatest diagnostic challenges in clinical medicine. The first association between cancer and CS was first reported in a patient in 1928 (1). However, it was only in the 1960s that ACTH production was demonstrated in tumours other than pituitary tumours (2). Following this, many malignancies other than small cell carcinoma of the lung (SCCL) were recognized to be causative for CS and in several large series, ectopic ACTH secretion has shown to account for approximately $10 \%$ of Cushing's syndrome (3). The causative malignancies include carcinoid tumours of the lungs, thymus and gastrointestinal tract, islet cell tumours, phaeochromocytomas and medullary thyroid carcinomas (4).

The syndrome requires a complete workup that includes the establishment of endogenous CS, diagnosis of ACTH dependency, localization of the source of ACTH secretion and rapid biochemical control of hypercortisolaemia. The effects of severe hypercotisolaemia make the diagnostic pathway difficult. Here, we describe a patient with ectopic ACTH from a bronchial carcinoid tumour highlighting the unusual presentation and difficulties in management.

\section{Case report}

A 50-year-old police woman presented to the medical casualty department with acutely worsening shortness of breath, chills, rigors and general deterioration. She admitted that she was having a 12 month history of facial swelling, weight gain, hair loss, fatigue, dyspnea with poor effort tolerance, depression and generalized ill health. She had also developed diabetes mellitus and hypertension 1 year prior to presentation with no family history of the same and had poor control despite being on treatment. She denied any history of taking exogenous steroids or herbal medicine.

On examination, she was noted to be extremely breathless, pigmented, hypertensive and centrally obese. She had a clinically Cushingoid appearance with central obesity, proximal myopathy, easy bruising, facial puffiness and fat deposition in the dorso-cervical region. She had purple stria over her flanks and thighs as well as facial acne and hyperpigmentation of her extensor surfaces on her upper and lower limbs (figure 1). Thyroid examination was normal.

Preliminary investigations revealed that she was hyperglycaemic and hypokalaemic (potassium 1.7

\footnotetext{
${ }^{1}$ Department of endocrinology, national hospital of Sri Lanka, ${ }^{2}$ Department of pathology, national hospital of Sri Lanka, ${ }^{3}$ Department of medicine, national hospital of Sri Lanka, ${ }^{4}$ Department of cardiothoracic surgery, national hospital of Sri Lanka.
} 
$\mathrm{mmol} / \mathrm{l})$. Although her inflammatory markers and white cell counts (WCC) were not suggestive of an infection, chest X-ray suggested a bilateral pneumonic state and blood cultures confirmed a growth of Staphylococcus aureas and she was treated for staphylococcal sepsis and pneumonia with intravenous antibiotics.

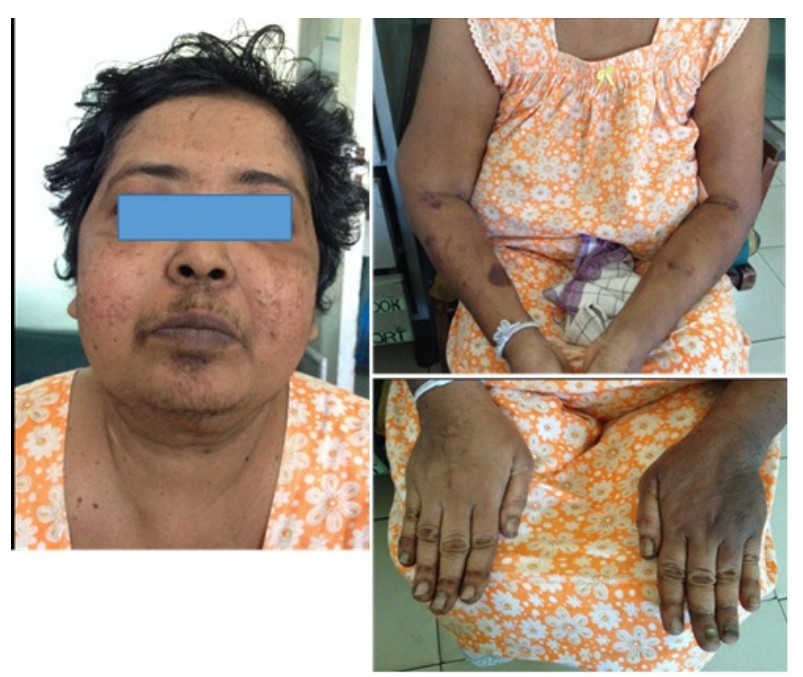

Figure 1.

Subsequently, she was evaluated for CS. Her 9 am plasma cortisol was unsuppressed after overnight dexamethasone at $890 \mathrm{nmol} / \mathrm{L}$ (reference: $<50 \mathrm{nmol} / \mathrm{L}$ ) and 9 am plasma cortisol after 48 hours of low dose dexamethasone was $792 \mathrm{nmol} / \mathrm{L}$ (reference: $<50 \mathrm{nmol} / \mathrm{L}$ ), which confirmed a diagnosis of CS. Her plasma ACTH was $212 \mathrm{pg} / \mathrm{ml}$ (reference $<50 \mathrm{pg} / \mathrm{ml}$ ). Thus an extensive search was planned to establish the source of ACTH. As she was very symptomatic, she was commenced on oral ketoconazole 200mg 12 hourly. MRI of the pituitary region reported a microadenoma, which was $1 \mathrm{~mm}$ in size, giving rise to the need for an inferior petrosal sinus sampling (IPSS) to localize the source of ACTH (figure 2).

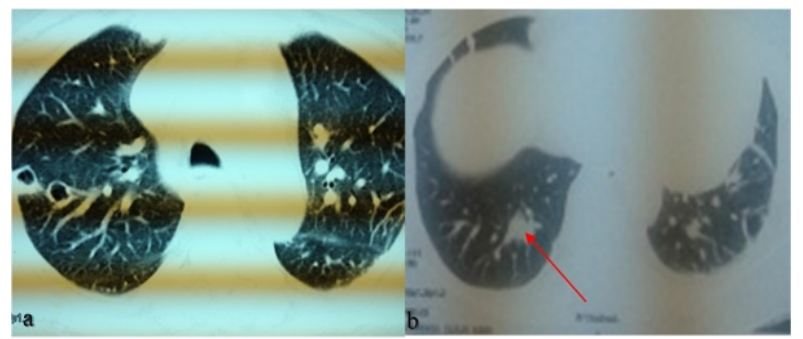

Figure 2.
Table 1.

\begin{tabular}{lcc}
\cline { 2 - 3 } & \multicolumn{2}{c}{ ACTH levels $(\mathrm{pg} / \mathrm{ml})$} \\
\hline & Right & Left \\
Inferior petrosal sinus & 113.1 & 119.6 \\
Internal jugular & 116.4 & 121.8 \\
peripheral & 108.2 & \\
\hline
\end{tabular}

Central ACTH level / peripheral ACTH level = 1.02 ( $<1.8$ - Cushing's disease unlikely)

She underwent a bilateral IPSS without corticotrophin releasing hormone $(\mathrm{CRH})$ stimulation. The results of the sampling clearly demonstrated that the source of ACTH was unlikely to be the pituitary as the ratio between central to peripheral ACTH levels was1.02.

Her clinical status was again compromised following the procedure where she developed a urinary tract infection and an abscess at the femoral puncture site. Her condition got progressed to septic shock and was resuscitated with fluids, inotropes and hydrocortisone therapy.

In the meantime, she had a CT scan of the chest, which revealed bilateral diffuse cavitatory lesions with patchy consolidation (figure 2). Interestingly, a single solid lesion in the right lower lobe was reported as a "nodule, which may represent metastatic deposit" and the radiology team suggested a CT guided biopsy. Even after this procedure, the patient became extremely breathless with type 1 respiratory failure. The $\mathrm{CT}$ findings were suggestive of early infective process on a background of recent staphylococcal pneumonia. Notably her ESR was $5 \mathrm{~mm} /$ 1 st hour and WCC were normal during this time. However, the clinical state was that of an infection and she was immediately treated with carbepenum as well as a systemic antifungals whilst awaiting cultures. Blood and sputum culture yielded extended-spectrum beta-lactamase (ESBL) organisms. As she was on ketoconazole, her cortisol reserve was insufficient to counteract the stress of severe infection and she received intravenous hydrocortisone with fluid resuscitation. As expected, her random blood cortisol done before steroid administration revealed a very low cortisol level of $151 \mathrm{nmol} / \mathrm{L}$. Therefore, diagnostic tests had to be postponed at this stage as she was not fit enough for invasive investigations.

Following recovery from the infection, the patient underwent another CT scan of the chest to re-evaluate the lung lesions. This revealed resolution of almost all the cavitatory lesions and consolidation. Interestingly, the small area of consolidation in the right lower lobe remained the same. CT scan of abdomen and pelvis revealed bilateral nodular hyperplasia of the adrenals as well as a lesion 
in the liver, which avidly enhanced with contrast. This was later confirmed to be a haemangioma by ultrasound assessment. Bronchoscopy did not reveal any endobronchial lesions. Octreotide scintigraphy or FDG-PET scan could not be performed due to non-availability.

A CT guided biopsy of her right lung nodule was performed and the histopathology revealed a tumour composed of nests of small cells with hyperchromatic nuclei having finely stippled chromatin. The mitotic count was low (2 per $10 \mathrm{hpf})$. Small, eosinophilic nucleoli were present staining strongly for ACTH, CD56, chromogranin and synaptophysin (figure 3).

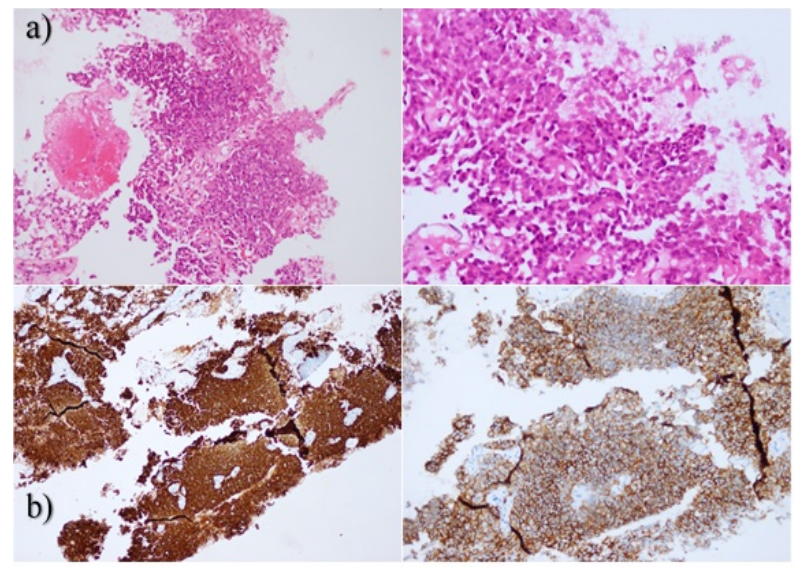

Figure 3.

These histological features are shared by SCLC as well as bronchial carcinoids. Differentiation of these 2 lesions can be done using the Ki-67 proliferation index, which would reveal a very low index for carcinoids whereas it would be a very high index for SCLC (figure 4).

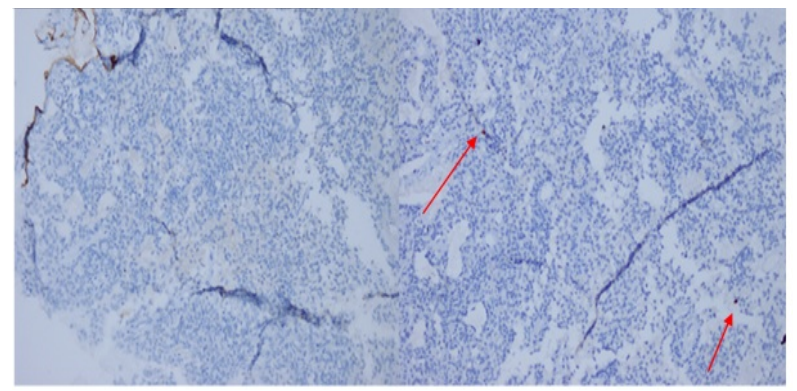

Figure 4.

Following the histological diagnosis of bronchial carcinoid, the patient underwent right lower lobectomy and she tolerated the procedure well. The resected specimen contained a $2.2 \times 2.2 \mathrm{~cm}$ size bronchial carcinoid, which was reconfirmed with histology. Following thoracotomy and resection of her lung lesion, the plasma cortisol decreased significantly (post op $25 \mathrm{nmol} / \mathrm{L}$ ) and she remains well on maintenance dose of steroids. When reviewed in clinic 3 months after surgery, she was symptomatically well, with good lung function.

\section{Discussion}

Bronchial carcinoid, which is a low to moderate grade malignancy, typically has a long history and slow onset of symptoms (1-84 months, median 23.6). Due to this slow progressive nature of this tumour, the classical Cushingoid features are usually present by the time they present with clinical symptoms attributable to lung disease (3). Our patient had symptoms of cortisol excess for at least 12 months prior to presentation. As in most cases, the tumour by itself did not give rise to respiratory symptoms or signs, but secondary to infections of the lung.

The investigative process and management of a patient with CS is very challenging. The hypercortisolaemia leads to hyperglycemia, fluid retention, hypertension, severe infections, electrolyte abnormalities and many other problems that undoubtedly hinder the investigative process. Additionally, these patients cannot mount an adequate cortisol response (even though they have very high basal levels of cortisol) in the event of stress such as sepsis or surgery. Therefore, these patients are always at risk of adrenal crisis, especially when they are on adrenolytic drugs such as ketoconazole, as in this patient. Adrenal insufficiency occurs insidiously and has very subtle features such as better control of blood sugar and blood pressure in a previously uncontrolled patient or reduction in drug doses to achieve control. Thus, daily bed side assessment for these features is mandatory in addition to testing for cortisol levels to prevent an adrenal crisis. This case also highlights the immunocompromised nature of this disease process and the need for extra vigilance in identifying and treating them with steroids appropriately. Our patient presented with a severe atypical pneumonia and continued to have recurrent infections during the investigative process. She also developed urinary sepsis and infection at the catheter site after IPSS. In all these infections, her CRP and ESR did not increase and if the desired clinical vigilance was not advocated, these infections would have not been picked up until in a very advanced state.

Confirmatory investigations for suspected CS in the presence of severe sepsis and critical illness may lead to falsely elevated cortisol levels. Thus, the workup and evaluation for possible hypercortisolism should not be performed while patients are under stress.

Differentiating Cushing disease from an ectopic source of ACTH can be difficult. Generally, these patients 
with ectopic secretion of ACTH have higher ACTH levels. They fail to suppress cortisol secretion for high doses of dexamethasone $(8 \mathrm{mg})$ and their pituitary adrenal responses to $\mathrm{CRH}$ stimulation is also absent $(5,6)$. However, $20-40 \%$ of patients with ectopic ACTH demonstrate cortisol suppression on high dose dexamethasone and $10-15 \%$ responds to $\mathrm{CRH}$ stimulation (6). Pituitary imaging may unmask incidental lesions and pose difficulties in the diagnosis. It is reported that, 10$20 \%$ of endocrinologically normal people have pituitary lesions of no clinical significance on pituitary imaging (7). Pituitary imaging can also lead to false negative results especially in the case of micro adenomas where dynamic imaging with contrast is not used. Modern MRI scanning has been reported to have only $70-80 \%$ sensitivities in the detection of micro adenomas (8).

The most valuable investigation for differentiating between pituitary and ectopic sources is the inferior petrosal venous sinus sampling (IPSS), which is considered as the gold standard. A baseline ACTH gradient between the inferior and the peripheral petrosal sinuses $>2$, and after stimulation with $\mathrm{CRH} /$ desmopressin $>3$, would indicate a pituitary source of ACTH secretion $(9,10)$. This is an invasive procedure, which need expert skills and may be difficult in a critically ill patients.

Once it is confirmed that the ACTH is from an ectopic source, the localization of the source of ectopic ACTH can be even more difficult, especially if standard imaging is negative. It is also important to consider the possibility of infections and unrelated benign incidental lesions, which may mislead the clinician if not interpreted in their true context. In our patient, the CT scan of the chest showed bilateral cavitatory lesions and the peripheral lung nodule. These findings along with the enhancing lesion in the liver could easily have been misdiagnosed as advanced malignancy. The index lesion in the lung was only made prominent after resolution of the florid inflammatory changes and in this situation, patience was the key to the diagnosis.

Confirmation of ectopic ACTH production requires demonstration of immunostaining positivity for ACTH in the resected tumour. Thereafter, the use of ki-67 marker is the key factor in differentiating SCLC from carcinoid tumour, as both these are entities are in a spectrum of neuroendocrine tumours.

Management of patients with ectopic ACTH requires control of the hypercortisolaemia as soon as the diagnosis is established (11). Ketoconazole and metyrapone have reasonable amount of evidence for their efficacy and safety $(12,13)$. Patients with identifiable sources of ectopic ACTH should have the tumours resected and surgery can offer a cure in more than $80 \%$ of bronchial carcinoids (12).
Even with modern techniques, in as many as $12 \%$ of patients, the source of ACTH may not be found (11). Such cases with "occult" ACTH-secreting tumours remain a challenge and may need repeated investigations for many years. In such cases, bilateral adrenalectomy is the next best therapeutic option, but with the need for lifelong steroid replacement (14).

\section{Conclusion}

Ectopic ACTH secreting tumours present some of the most challenging differential diagnoses in endocrinology and require careful clinical, biochemical, radiological, and pathological investigation. Due vigilance for sepsis, adrenal insufficiency and other complications of high cortisol is needed and close liaison between the endocrinologist, endocrine surgeon, chemical pathologist, and radiologist in the management of these patients is the key for a successful outcome.

\section{References}

1. Brown W H, "A case of pluriglandular syndrome: "diabetes of bearded women", The Lancet 1928; 212: 1022-3.

2. Liddle GW, Island DP, Ney RL, Nicholson W E, Shimizu N, "Non pituitary neoplasms and Cushing's syndrome. Ectopic "adrenocorticotropin" produced by non pituitary neoplasms as a cause of Cushing's syndrome," Archives of Internal Medicine 1963; 111; 471-5.

3. Beuschlein Fand Hammer GD, "Ectopic pro-opiomelanocortin syndrome," Endocrinology and Metabolism Clinics of North America 2002; 31(1); 191-234.

4. Aniszewski JP, Young WF, Thompson GB, Grant CS, Van Heerden JA. "Cushing syndrome due to ectopic adrenocorticotropic hormone secretion," World Journal of Surgery 2001; 25(1-7); 934-40.

5. Ilias, Torpy DJ, Pacak K, Mullen N, Wesley RA, Nieman LK "Cushing's syndrome due to ectopic corticotropin secretion: twenty years' experience at the National Institutes of Health," Journal of Clinical Endocrinology and Metabolism 2005; 90(8): 4955-62.

6. Howlett TA, Drury PL, Perry L, Doniach I, Rees LH, Besser GM. "Diagnosis and management of ACTHdependent Cushing's syndrome: comparison of the features in ectopic and pituitary ACTH production," Clinical Endocrinology1986; 24: 699-713.

7. Kaye TB, Crapo L. The Cushing's syndrome: an update on diagnostic tests. Ann Intern Med 1990; 112: 434-44.

8. Herder WW, Uitterlinden P, Pieterman H, et al. Pituitary tumour localization in patients with Cushing's disease by magnetic resonance imaging. Is there a place for petrosal sinus sampling Clin Endocrinol (Oxf) 1994; 40: 87-92.

9. Kaltas GA, Giannuli MG, Newell-price JDC, et al. "A critical analysis of the value of simultaneous inferior petrosal sinus sampling in Cushing's disease and the occult ectopic adrenocorticotropin syndrome," The Journal of Clinical Endocrinology \& Metabolism 1999; 84: 487-92. 
10. Lefournier V, Martinie M, Vasdev A, et al. Accuracy of bilateral inferior petrosal or cavernous sinuses sampling in predicting the lateralization of Cushing's disease pituitary microadenoma: influence of catheter position and anatomy of venous drainage. JCEM 2003; 88: 196-203.

11. Isidori $\mathrm{M}$, Kaltas GA, Pozza $\mathrm{C}$, et al. "The ectopic adrenocorticotropin syndrome: clinical features, diagnosis, management and long-term follow-up" The Journal of Clinical Endocrinology \& Metabolism 2006; 91, 371-7.

12. Tabarin, A. Navarranne J. Guerin, J. B. Corcuff, M. Parneix, and P. Roger, "Use of ketoconazole in the treatment of
Cushing's disease and ectopic ACTH syndrome," Clinical Endocrinology 1991; 34(1); 63-9.

13. Engelhardt D and Weber MM, “Therapy of Cushing's syndrome with steroid biosynthesis inhibitors," Journal of Steroid Biochemistry and Molecular Biology 1994: 49 (4-6), 261-7.

14. Salameh JR, Borman KR, and Varkarakis GM, "Laparoscopic bilateral adrenalectomy for occult ectopic ACTH syndrome," Journal of Laparoendoscopic and advanced Surgical Techniques 2008; 18(1): 52-5. 